\title{
La Asociación Latinoamericana de Tórax Una oportunidad para el desarrollo científico de la medicina respiratoria de la región
}

El mundo actual no permite concebir el desarrollo en ningún área del conocimiento sin el trabajo en equipo, en muchos casos interdisciplinario e intersectorial, a través de redes de información, intercambio científico y trabajo colaborativo. La salud y la enfermedad respiratoria no son la excepción.

Es cierto que Latinoamérica incluye países y regiones con diferencias sensibles en todos los campos, pero los grandes equipos no se conforman de iguales sino, por el contrario, de diferentes y complementarios, cada cual con su fortaleza y su capacidad de aportar. Por otra parte, la unidad en el idioma, español y portugués, y una forma particular de ver la vida nos acerca en la posibilidad de crecer juntos.

Es esta la gran oportunidad que nos ofrece la Asociación Latinoamericana de Tórax - ALAT - como opción para el desarrollo del conocimiento, en su generación, divulgación y aplicación, en beneficio de la salud respiratoria de nuestra población y de la calidad de vida de nuestros enfermos.

La madurez que ha alcanzado la ALAT, una asociación científica que agrupa profesionales con interés en la salud y la enfermedad respiratorias en Latinoamérica, en algo más de 15 años, le da capacidad para ampliar el cumplimiento de su misión fundamental que, dado su carácter científico, es la educación continuada y la investigación en el campo de la neumología y la cirugía del tórax usando como estrategia principal la creación de canales abiertos de comunicación y de una red de centros de información, entrenamiento e investigación que permitan el crecimiento científico de todos sus miembros en pro de la salud respiratoria de la región.

\section{UN VISTAZO A SU CREACIÓN Y CRECIMIENTO}

La ausencia de un evento internacional de alto nivel científico en el área de la neumología y la cirugía del tórax que congregara a todos los países de habla castellana y portuguesa, motivó a los doctores Roberto RodríguezRoisin de Barcelona y Manuel Cosio y Alejandro Grassino de Montreal, a organizar lo que sería el I Congreso Iberoamericano de Neumología, el cual se realizó en Barcelona en 1990.

La idea venía madurándose de tiempo atrás gracias al interés de un grupo de neumólogos latinoamericanos formados en Norteamérica y Europa, como Bartolomé Celli, Jacob lasha Snajder y José Jardim, y cuya identidad en el idioma los acercó a un grupo de españoles encabezados por Rodríguez-Roisin. Vino la consolidación con el II Congreso Iberoamericano en Acapulco, Méjico, en 1992, liderado por Moisés Selman y el III Congreso en Viña del Mar, Chile, en 1994, presidido por Rodrigo Moreno.

Más allá de la identidad en el idioma, la conciencia de la identidad cultural y la similitud de nuestra problemática y oportunidades, sustentó la necesidad del trabajo conjunto y el apoyo mutuo a través de una asociación científica, que al igual que las Sociedades Americana de Tórax (ATS) y la Europea de Enfermedades Respiratorias (ERS), impulsara firmemente el desarrollo de la neumología en Latinoamérica dentro de los más altos niveles de calidad científica. Con este propósito, en el Congreso de la ATS en mayo de 1996, se nombró una comisión coordinada por José R. Jardim para trabajar los estatutos y, finalmente, se dio vida a la Asociación Latinoamericana del Tórax - ALAT-en noviembre de 1996 durante el IV Congreso Iberoamericano de Neumología en Caracas, designándose como su primer presidente a José R. Jardim, de Brasil, quien imprimió a la ALAT ese primer impulso. Bajo su dirección, se realizó con éxito, el I Congreso de la ALAT en la ciudad de Sao Paulo, Brasil. 
Con estilos y dinámicas diferentes pero complementarias, cada uno de los presidentes, Juntas y Consejos Directivos que se han sucedido desde entonces, ha impulsado el desarrollo administrativo y científico de nuestra asociación. Algunos hechos, no todos, que han marcado ese desarrollo han incluido:

- El acercamiento con la Unión Latinoamericana de Sociedades de Tisiología y Enfermedades Respiratorias - ULASTER - para darle unidad al trabajo bajo el enfoque de asociación científica de miembros individuales como lo es la ALAT.

- $\quad$ La creación y crecimiento de los Departamentos Científicos

- $\quad$ La consolidación de la estructura definida estatutariamente y su funcionamiento

- $\quad$ La organización administrativa y financiera

- La consolidación de un Congreso periódico de la ALAT con posibilidad de realizarse de manera autónoma: Cartagena 2000, Montevideo 2012 y en adelante.

- $\quad$ La trascendente alianza con la Sociedad Española de Neumología (SEPAR) que posibilitó que la Revista Archivos de Bronconeumología fuera también la revista oficial de la ALAT y la institución de las becas SEPAR ALAT que han beneficiado a un grupo muy significativo de profesionales latinoamericanos.

- La Alianza con las sociedades científicas transnacionales más importantes: ATS, ERS, ACCP, APSR y el posicionamiento de la ALAT como la representante de la neumología latinoamericana ante la OMS, la Unión Internacional contra la Tuberculosis y las Enfermedades Respiratorias (UICTER), el Foro de Sociedades Respiratorias Internacionales (FIRS), entidades gubernamentales y agrupaciones científicas como GOLD y GINA.

- El desarrollo académico y científico de la ALAT, lo más importante, como fin último de la asociación, plasmado en la calidad creciente de sus eventos y de las investigaciones y publicaciones de sus miembros, cuya más clara expresión es el proyecto latinoamericano de investigación en enfermedad obstructiva - PLATINO -, y los cursos itinerantes en EPOC y entrenamiento en espirometría asma y sueño.

- $\quad$ La creación de una Red de Centros de Investigación y entrenamiento en Latinoamérica - REDINLAT-, cuyas bases se han definido.

- $\quad$ La creación de una publicación periódica "Newsletter" de ALAT - ULASTER que se ha ido consolidando.

El estudio PLATINO y los cursos itinerantes como expresión de la capacidad científica de la neumología latinoamericana. El Proyecto Latinoamericano de Investigación en Obstrucción Pulmonar es una iniciativa de la ALAT, cuyo objetivo es estudiar la prevalencia de la Enfermedad Pulmonar Obstructiva Crónica y sus principales factores de riesgo en cinco grandes áreas metropolitanas de América Latina. Ha generado más de 35 publicaciones y ha iniciado una segunda fase: PLATINO II. Este es un ejemplo de trabajo colaborativo.

Los cursos itinerantes de EPOC, cerca de 30, han llegado a prácticamente todos los países latinoamericanos con una altísima calidad científica y un impacto real por su metodología lo mismo que los cursos de entrenamiento en espirometría.

\section{PRESIDENTES DE ALAT}

- Jose R. Jardim (Brasil)

- Carmen Lisboa (Chile)

- Carlos Torres-Duque (Colombia)

- Carlos Luna (Argentina)

- Rogelio Pérez-Padilla (Méjico)

- Mauro Zamboni (Brasil)
$1996-2000$
$2000-2002$
$2002-2004$
$2004-2006$
$2006-2008$
$2008-2010$ 
- María Victorina López (Uruguay) 2010 - 2012

- María Montes de Oca (Venezuela) 2012 - 2014

\section{CONGRESOS DE LA ALAT}

$\begin{array}{lll}\text { - I Congreso } & \text { Sao Paulo, Brasil } & 1998 \\ \text { - II Congreso } & \text { Cartagena, Colombia } & 2000 \\ \text { - III Congreso } & \text { Punta del Este, Uruguay } & 2002 \\ \text { - IV Congreso } & \text { Buenos Aires, Argentina } & 2004 \\ \text { - V Congreso } & \text { Cancún, Méjico } & 2006 \\ \text { - VI Congreso } & \text { Brasilia, Brasil } & 2008 \\ \text { - VII Congreso } & \text { Lima, Perú } & 2010 \\ \text { - VIII Congreso } & \text { Montevideo, Uruguay } & 2012\end{array}$

\section{UN FUTURO PROMISORIO, COLOMBIA INVITA AL COMPROMISO}

La medicina respiratoria colombiana, representada en la Asociación Colombiana de Neumología y Cirugía de Tórax y en cada uno de sus miembros, ha tenido un papel activo en la ALAT que pretende ampliar en busca del desarrollo regional. Más allá de mi presidencia (2002 - 2004), en la cual buscamos fortalecer la comunicación, dinamizar las estructuras científicas de la asociación definidas estatutariamente y durante la cual creamos REDINLAT, impulsada por Alejandro Casas, el país organizó el II Congreso de ALAT en Cartagena (2000) presidido por Darío Maldonado, y ha participado activamente en los Departamentos Científicos, presidiendo en su momento algunos de ellos: Alejandro Casas (EPOC), Hugo Caballero (Endoscopia) y Stella Martínez (Cirugía). La presencia de Alejandro Casas en las actividades y documentos de la iniciativa GOLD fue sobresaliente.

La enfermedad respiratoria es muy prevalente y desconocemos en gran medida sus características clínicas y epidemiológicas para enfrentarla más apropiadamente. El ejemplo del proyecto PLATINO y la madurez que está alcanzando la ALAT son un estímulo para la aceleración del desarrollo de la medicina respiratoria en Latinoamérica.

Contamos con profesionales, instituciones y agrupaciones científicas con altos de estructura, recursos, tecnología, logística y organización científica y con la posibilidad de articularlos para el desarrollo de investigación y entrenamiento de calidad alta en medicina respiratoria y planes de trabajo conjunto en beneficio de los miembros de la ALAT y de la salud respiratoria de la población.

La cita es en el VIII Congreso de la ALAT en Montevideo en la fase final de la presidencia de María Victorina López, en cuyo período, la Asociación ha tenido un dinamismo admirable, en todos sus aspectos, científico y administrativo. Una felicitación muy grande para ella y todo su equipo de trabajo por esta labor. Es un momento único para que todos seamos miembros y participemos activamente en la Asociación.

Carlos Arturo Torres Duque Director, Departamento de Investigación y Educación Fundación Neumológica Colombiana Ex presidente de la ALAT 